\title{
Rat Monoclonal Antibodies Specific for LST1 Proteins
}

\author{
Christian Schiller, ${ }^{1,2}$ Maximilian J.E. Nitschké, Alexander Seidl, ${ }^{1}$ \\ Elisabeth Kremmer, ${ }^{2}$ and Elisabeth $\mathrm{H}$. Weiss ${ }^{1}$
}

The LST1 gene is located in the human MHC class III region and encodes transmembrane and soluble isoforms that have been suggested to play a role in the regulation of the immune response and are associated with inflammatory diseases such as rheumatoid arthritis. Here we describe the generation and characterization of the first monoclonal antibodies against LST1. Two hybridoma lines secreting monoclonal antibodies designated 7E2 and 8D12 were established. The 7E2 antibody detects recombinant and endogenous LST1 by Western blot analysis while 8D12 reacts with recombinant and endogenous LST1 in immunoprecipitation and flow cytometry procedures. The newly established antibodies were used to survey LST1 protein expression in human cell lines, which was found to be tightly regulated, allowing the expression of transmembrane isoforms but suppressing soluble isoforms.

\section{Introduction}

T HE MHC CLASS III REgION encodes numerous genes involved in the inflammatory response and harbors the LST1 (leukocyte specific transcript 1) gene. ${ }^{(1)}$ LST1 transcripts are strongly present in immune cells, especially B cells, T cells, monocytes, and dendritic cells, ${ }^{(2)}$ and their expression has been shown to be drastically upregulated by inflammatory stimuli. ${ }^{(3,4)}$ LST1 transcripts undergo extensive alternative splicing, resulting in 17 splice variants that encode 12 transmembrane or soluble isoforms. Five distinct transcriptional start sites have been identified in the LST1 gene. ${ }^{(5)}$ The alternative exon 1 sequences result in transcripts differing in the $5^{\prime}$-untranslated region $\left(5^{\prime}\right.$ UTR). Thus, translational control of LST1 mRNAs through the 5'-UTR may represent an additional mechanism to regulate LST1 expression in immune cells. All LST1 splice variants contain the exon 2 sequence, which harbors the common translation initiation site, but vary with regard to the presence of exon 3 and exon 4 , respectively, and in the length of the last exon 5. Transcripts lacking the transmembrane-helix-encoding exon 3 code for short soluble polypeptides, whereas transcripts lacking exon 4 undergo a translational frameshift, which results in a premature stop codon and an alternative carboxyterminal amino acid sequence. Finally, two additional splice sites are present in the exon 5 sequence (exon $5^{\prime} /$ exon $5^{\prime \prime}$ ), further adding to isoform diversity. Here we name the different LST1 isoforms based on the protein coding exons present in the respective transcript but also specify the letter designations ${ }^{(4,6)}$ (Fig. 1).

It has been proposed that LST1 isoforms play an important role in regulating the immune response ${ }^{(6)}$ and enabling cell-cell communication, ${ }^{(7)}$ but the exact mechanisms involved are yet to be elucidated. Recent work has also shown that high LST1 expression levels are associated with several inflammatory diseases including rheumatoid arthritis $^{(4)}$ and the inflammatory response believed to cause neural tube defect. ${ }^{(8)}$ These associations, however, have been established based on mRNA expression data and have not been validated at the protein level. To allow insights into the structure and function of LST1 isoforms and to discern their potential role in autoimmune diseases, we generated and characterized rat monoclonal antibodies specific for LST1.

\section{Experimental Procedures}

\section{Isolation and detection of LST1 transcripts}

Total RNA was isolated from human U-937 cells using the Trizol reagent (Invitrogen, Paisley, United Kingdom). Transcripts were amplified using the One Step RT-PCR Kit (Qiagen, Hilden, Germany) following the manufacturer's instructions and using primers flanking the longest open reading frame of LST1 (5-Lst2s: 5'-GCAAGGACTAGAGTTCCTGAC-3'; 3-Lst1A: 5'-CGGATCCTTATCAGGTGGGTTTGTTCTC-3'). Amplification products were separated on $2 \%$ agarose gels, together with a molecular weight marker $(1 \mathrm{~Kb}$ Plus DNA Ladder, Invitrogen).

\section{Cloning of a HIS-LST1 expression construct}

The HIS-LST1.3 $\Delta 45^{\prime}$ construct was cloned by amplifying the mutated LST1.3 $\Delta 45^{\prime}$ cDNA (by exon usage: $3 \Delta$ [lacking

\footnotetext{
${ }^{1}$ Department of Biology II, Ludwig-Maximilians-Universität München, Germany.

${ }^{2}$ Institute of Molecular Immunology, Helmholtz Zentrum München, Germany.
} 
the sequence encoding the transmembrane helix], 4 and $5^{\prime}$ ) using the following primers: 5-Lst3-Bgl: 5'-CAGATCTAT CCGCCTGCCTGTGTTG-3' ${ }^{\prime}$ and 3-Lst1A as described above. The LST1.3 $\Delta 45^{\prime}$ PCR product was subcloned into the pCRII vector using the TOPO TA cloning kit (both from Invitrogen) and subsequently cloned into the pET-45b (+) vector (Novagen, Gibbstown, NJ).

\section{Generation of monoclonal rat anti-LST1 antibodies}

Recombinant HIS-LST1 was generated by transforming the HIS-LST1.3 $\Delta 45^{\prime}$ vector into BL21 Codon Plus (DE3)-RIPL bacteria (Stratagene, La Jolla, CA) and induction of protein expression by treatment with $1 \mathrm{mM}$ IPTG (Carl Roth, Karlsruhe, Germany) for $3 \mathrm{~h}$ while shaking at $37^{\circ} \mathrm{C}$. Bacterial extracts were prepared with lysis buffer $(50 \mathrm{mM}$ sodium phosphate buffer [pH 7.4], $300 \mathrm{mM} \mathrm{NaCl}, 0.2 \mathrm{mg} / \mathrm{mL}$ lysozyme, and complete protease inhibitor cocktail; Roche Diagnostics, Mannheim, Germany) for $30 \mathrm{~min}$ at $4^{\circ} \mathrm{C}$. Subsequently lysates were adjusted to $2 \mathrm{M}$ urea and sonicated using a Sonifier 250 (Branson, Pusan, Korea). To remove unsoluble debris, lysates were spun at $40,000 \mathrm{~g}$ at $4{ }^{\circ} \mathrm{C}$ for $45 \mathrm{~min}$ followed by filtration of supernatants through a $0.2 \mu \mathrm{m}$ pore sterile filter. Purification of HIS-LST1 from bacterial extracts was achieved by affinity chromatography using ProPur IMAC Midi columns (Nunc, Langenselbold, Germany) following the manufacturer's recommendations. Approximately $50 \mu \mathrm{g}$ of the purified HIS-LST1-fusion protein were injected both intraperitoneally (i.p.) and subcutaneously (s.c.) into Lou/C rats using CPG2006 (TIB MOLBIOL, Berlin, Germany) as adjuvant. After an 8-week interval, a final i.p. and s.c. boost was given 3 days before fusion. Fusion of the rat spleen cells was performed with the myeloma cell line P3X63-Ag8.653. Hybridoma supernatants were tested in a solid-phase immunoassay. HIS-LST1-fusion protein was coated overnight at a concentration of $3 \mu \mathrm{g} / \mathrm{mL}$. After blocking with non-fat milk, hybridoma supernatants were added. Bound rat monoclonal antibodies (MAbs) were detected with a cocktail of biotinylated mouse MAbs against the rat IgG heavy chains ( $\alpha$-IgG1, $\alpha$-IgG2a, $\alpha$-IgG2b from ATCC, Manassas, VA; $\alpha$-IgG2c, Ascenion, Munich, Germany), thus avoiding IgM MAbs. The biotinylated MAbs were visualized with peroxidase-labeled Avidin (Alexis, Gruenberg, Germany) and o-phenylenediamine as chromogen in the peroxidase reaction. Clones LST1 7E2 of rat IgG2b subclass and LST1 8D12 of IgG2a subclass were stably subcloned and used for further analysis.

\section{Cell culture and transfection procedures}

In this study the following human cell lines were used: the pancreatic adenocarcinoma cell line Capan-1 (ATCC HTB-79), the embryonic kidney cell line HEK 293T (ATCC CRL-11268), the cervix adenocarcinoma cell line HeLa 229 (ATCC CCL2.1), the colorectal adenocarcinoma cell line HT-29 (ATCC HTB-38), the acute T cell leukemia line Jurkat (ATCC TIB-152), the chronic myelogenous leukemia cell line K-562 (ATCC CCL-243), the malignant melanoma cell line SK-MEL-28 (ATCC HTB-72), the glioblastoma cell line U-251 MG (ATCC HTB-17), and the histiocytic lymphoma cell line U-937 (ATCC CRL-1593.2). Cell lines were cultivated as recommended by ATCC. HEK 293T cells were transiently transfected as described previously. ${ }^{(9)}$ U-937 stable transfectants were established by electroporation using an Electro Cell Manipulator
600 (BTX, Holliston, MA), with the following settings: $175 \mathrm{~V}$, $1300 \mu \mathrm{F}, 129 \Omega$. Negative selection was achieved by cultivating transfectants in growth medium containing $2.5 \mathrm{mg} / \mathrm{mL} \mathrm{G418}$ (PAA, Pasching, Austria) $48 \mathrm{~h}$ after electroporation. Clones were screened by FACS and Western blot analysis.

\section{Flow cytometry}

For flow cytometry, cells were washed and resuspended in PBS containing 5\% FCS. Staining was performed with the LST1 8D12 antibody (undiluted hybridoma supernatant) and a PE-conjugated goat anti-rat antibody (1:50, Dianova, Hamburg, Germany). After staining, cells were fixated in 1\% PFA/PBS. For intracellular staining, cells were fixated in $4 \%$ PFA/PBS prior to staining and antibody dilutions were supplemented with $0.1 \%$ Saponin. For each staining, 10,000 cells were analyzed using a FACSCalibur cytometer and Cell Quest software (both from Becton Dickinson, Franklin Lakes, NJ).

\section{Immunoprecipitation and Western blot analysis}

Immunoprecipitation was performed with either $10^{7}$ HEK 293 T or $3 \times 10^{7}$ U-937 cells. Cell lysates were prepared with lysis buffer (50 mM Tris- $\mathrm{HCl}[\mathrm{pH} 8.0], 150 \mathrm{mM} \mathrm{NaCl}, 1 \%$ Triton X-100, and complete protease inhibitor cocktail) for $30 \mathrm{~min}$ at $4^{\circ} \mathrm{C}$ followed by centrifugation at $13000 \mathrm{~g}$ and $4^{\circ} \mathrm{C}$ for $30 \mathrm{~min}$ to clear the lysate. Lysates were rolled at $4^{\circ} \mathrm{C}$ overnight with the LST1 8D12 antibody (100 $\mu \mathrm{L}$ hybridoma supernatant). The antibody-antigen complexes were subsequently coupled to $20 \mu \mathrm{L}$ Protein G Sepharose beads $(50 \% \mathrm{v} / \mathrm{v}$, Amersham, Buckinghamshire, UK) by rolling for $1 \mathrm{~h}$ at $4{ }^{\circ} \mathrm{C}$. Beads were washed with lysis buffer and stored in SDS sample buffer (Roti-Load 1, Roth) at $-20^{\circ} \mathrm{C}$. Western blot analysis was performed by boiling either the beads or cell lysates from $10^{6}$ cells in SDS sample buffer followed by separation on a $15 \%$ polyacrylamide gel; additionally $1 \mu \mathrm{L}$ of the prestained molecular weight marker Broad Range (Cell Signaling, Danvers, MA) was loaded onto each gel. Proteins were blotted overnight onto an Immobilion-FL PVDF membrane (Millipore, Schwalbach, Germany). To detect recombinant LST1, either the LST1 7E2 antibody (1:100, hybridoma supernatant) or the FLAG M2 antibody (1:1000, Sigma-Aldrich, Taufkirchen, Germany) was used. Endogenous LST1 was detected with the 7E2 antibody (1:10, hybridoma supernatant). Fluorescence detection was conducted with IRDye800coupled goat anti-mouse and goat anti-rat antibodies (1:10,000, Rockland, Gilbertsville, PA). For blot imaging, the Odyssey infrared imaging system (Li-cor, Lincoln, NE) was used.

\section{Results}

\section{Detection of recombinant and endogenous LST1 via Western blot analysis}

To ascertain whether the rat monoclonal 7E2 antibody detects different LST1 isoforms, recognition of several N- and C-terminally FLAG-tagged LST1 isoforms produced by transiently transfected HEK 293 T cells was assayed via Western blot analysis. The LST1 7E2 antibody clearly detected the transmembrane FLAG-LST1.2345' isoform (by exon usage of the coding transcript 2,3,4, and $5^{\prime}$ ) and its multimers but none of its C-terminally truncated degradation products (Fig. 1A, 
upper left panel, lane 1). Additionally the soluble FLAGLST1.245 isoform was clearly bound by the antibody (Fig. 1A, upper left panel, lane 5). Carboxyterminally tagged LST1.2345'-FLAG was not recognized, but a slightly truncated form lacking the C-terminal FLAG-tag (Fig. 1A, upper left panel, lane 2) was detected, indicating that the presence of the tag at the C-terminus interferes with antibody binding. Moreover, 7E2 did not react with the frame-shifted LST1.235' isoform, regardless of the location of the FLAG tag (Fig. 1A, upper left panel, lanes 3 and 4 ). The epitope recognized by the 7E2 antibody was determined with a mutant construct (FLAG-LST1.2345 $\Delta$ lacking the last $13 \mathrm{C}$-terminal amino acids

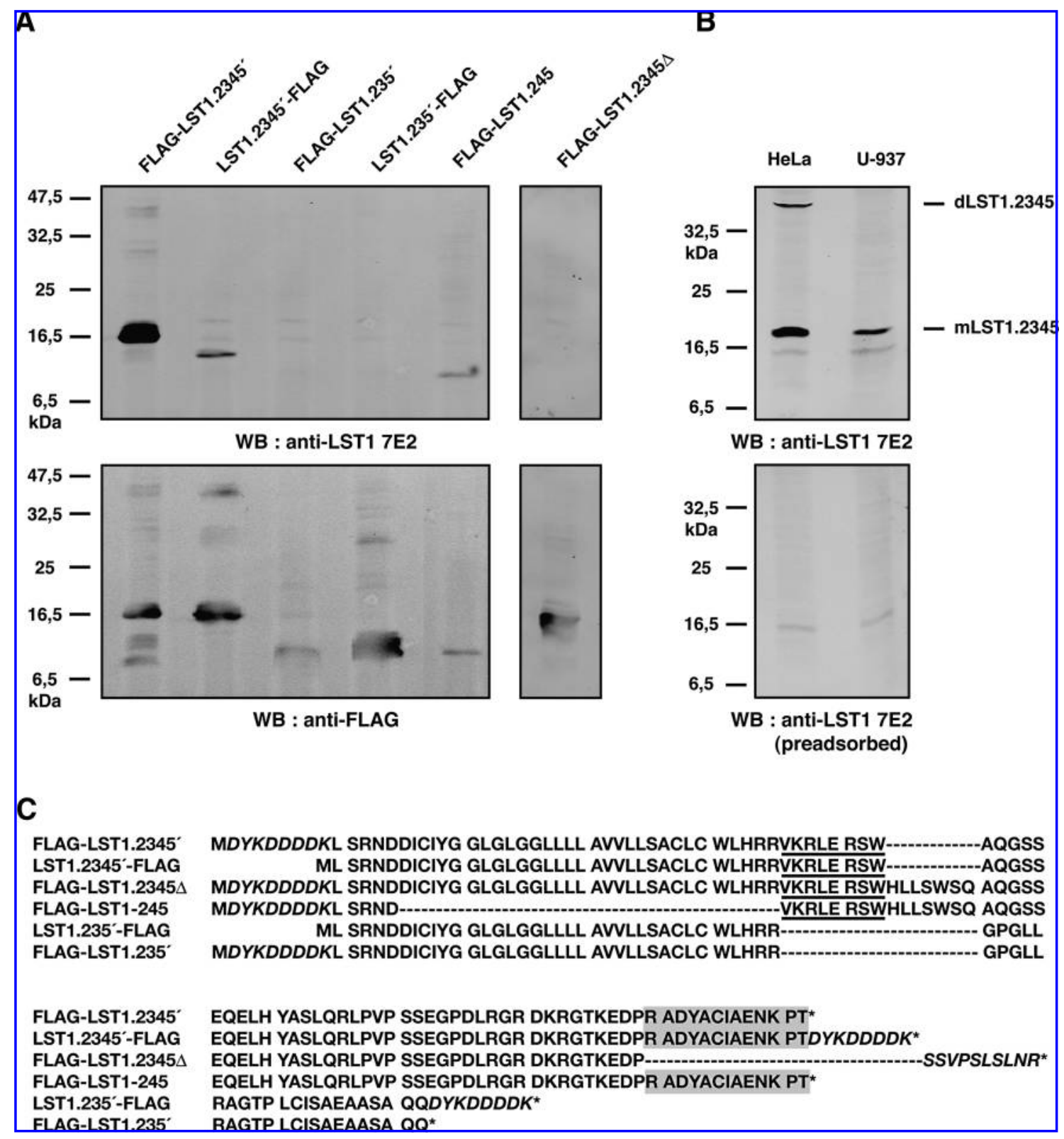

FIG. 1. Specificity of the rat monoclonal LST1 antibody 7E2. (A) Detection of recombinant LST1 by Western blot analysis. HEK 293T cells were transiently transfected with constructs encoding either N- or C-terminally FLAG-tagged LST1 $\left(\mathrm{LST} 1.2345=\mathrm{LST} 1 / \mathrm{P} ; \mathrm{LST} 1.2345^{\prime}=\mathrm{LST} 1 / \mathrm{ABG}\right.$; LST1.235' = LST1/K; LST1.245 = LST1/J). Lysates from transfectants were probed by Western blotting using the rat monoclonal LST1 antibody 7E2 (upper panels). Subsequently blots were stripped and reprobed with the FLAG specific antibody (lower panels). (B) Detection of endogenous LST1 by Western blot analysis. Lysates from HeLa and U-937 cells were probed by Western blotting using either the LST1 7E2 antibody (upper panel) or a HIS-LST1-pre-adsorbed LST1 7E2 antibody (lower panel). The position of the detected isoform LST1.2345 in its monomeric (mLST1) and dimeric (dLST1) form is indicated. (C) Binding epitope of the LST1 7E2 antibody. Amino acid sequence comparison of the recombinant LST1 isoforms probed in A. The amino acids encoded by exon 4 in the respective transcripts are underscored. The FLAG tag and the vector-encoded carboxyterminus of the truncated LST1.2345 $\Delta$ polypeptide are italicized. The epitope recognized by the 7E2 antibody is highlighted in grey. 
of full-length transmembrane LST1.2345. The LST1 7E2 antibody was unable to detect the truncated polypeptide (Fig. 1A, upper right panel). This allowed us to identify the 7E2 epitope in the C-terminal region of full-length LST1 (highlighted in Fig. 1C) and to conclude that the 7E2 antibody can detect all LST1 isoforms encoded by exon 4 containing splice variants.

Endogenous LST1 was detected by the 7E2 antibody in lysates from U-937 and HeLa cells. Only the full-length transmembrane LST1.2345 isoform (calculated molecular weight of $11.7 \mathrm{kDa}$ ) was observed as a monomer and as a possible homodimer (Fig. 1B). The reduced mobility of LST1 polypeptides in polyacrylamide gel electrophoresis has been previously observed. ${ }^{(7)}$ Antibody specificity was ensured by pre-adsorbing the 7E2 antibody with recombinant HISLST1 prior to immune detection, which failed to detect endogenous LST1 (Fig. 1B).

\section{Detection of native LST1 via immunoprecipitation and flow cytometry procedures}

The ability of the rat monoclonal LST1 8D12 antibody, which poorly recognized LST1 isoforms in Western blot analyses, to bind native LST1 was tested by immunoprecipitating recombinant FLAG-LST1.2345 and FLAG-LST1.245 from lysates of transiently transfected HEK 293T cells. The 8D12 antibody precipitated both transmembrane FLAG-LST1.2345' and soluble FLAG-LST1.245 (Fig. 2A, left panel). The 8D12 antibody also precipitated endogenous LST1.2345 from lysates of U-937 cells (Fig. 2A, right panel). Furthermore, using flow cytometry, the 8D12 antibody detected endogenous LST1 in
U-937 cells by intracellular staining. To assure binding specificity, a stable U-937 transfectant producing LST1.2345'FLAG was analyzed and revealed higher LST1 expression than that of untransfected U-937 cells (Fig. 2B).

\section{LST1 expression in human cell lines underlies tight regulation}

In previous studies we detected LST1 mRNA expression in a number of tissues and cells. Transcripts predominated in immune cells and the number of different splice variants found in these cells was higher than in cells from non-lymphoid tissue. ${ }^{(2)}$ Using the LST1 7E2 antibody, we found LST1 protein expression in cell lines of hematopoetic origin (U-937, $\mathrm{K}-562$, Jurkat) but also in cell lines originating from diverse tissues (Fig. 3A). Only a polypeptide corresponding to the full-length isoform LST1.2345 was detected as a monomer and as a possible homodimer. Even in U-937 cells, which express different LST1 transcripts, including the splice variant encoding the soluble isoform LST1.245' (Fig. 3B), only the fulllength polypeptide was detected. In contrast to mRNA results, LST1 protein levels were not higher in cells of immune origin nor were additional isoforms detected in these cells (Fig. 3A). Variability between the assayed cell lines was solely based on the distribution of LST1.2345 between its monomeric and dimeric form. Repeated efforts to detect the main soluble isoforms LST1.245/5' in a number of cell lines were unsuccessful (data not shown) and lead us to speculate that expression of LST1 is tightly regulated at the translational or post-translational level, resulting in the selective expression of

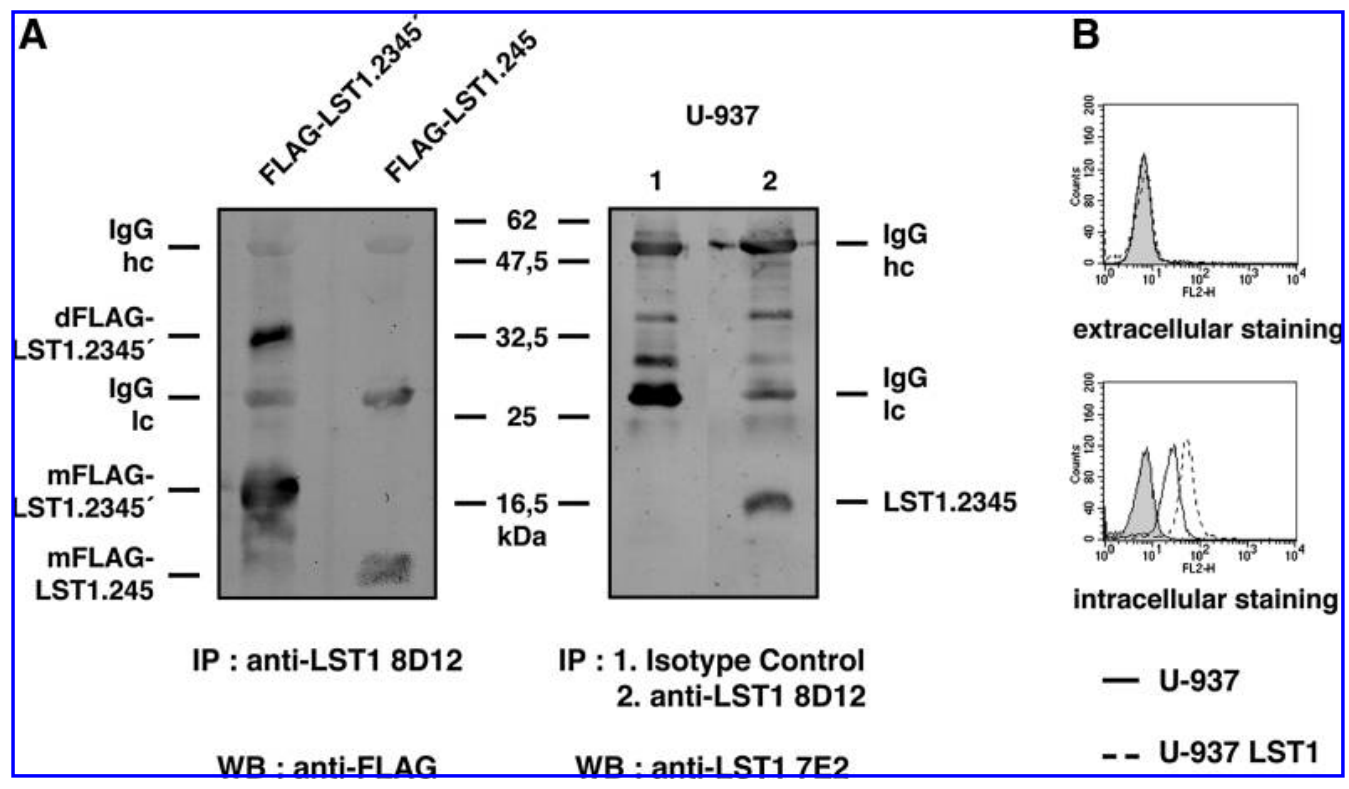

FIG. 2. Reactivity of the rat monoclonal LST1 antibody 8D12. (A) Immunoprecipitation of recombinant and endogenous LST1. HEK 293T cells were transiently transfected with constructs encoding either transmembrane FLAG-LST1.2345' or soluble FLAG-LST1.245. Recombinant FLAG-LST1 was precipitated using the LST1 8D12 antibody. Precipitates were probed via Western blot using the FLAG antibody (left panel). Endogenous LST1 was precipitated from U-937 lysates with either the LST1 8D12 or a rat control antibody of the same isotype. Precipitates were probed by Western blot using the LST1 7E2 antibody (right panel). The positions of recombinant and endogenous LST1 isoforms and of IgG light (lc) and heavy (hc) chains are marked. (B) FACS analysis of LST1 expression. U-937 cells (continuous line) and a stable U-937 LST1.2345'-FLAG transfectant (dotted line) were stained directly (extracellular) or after permeabilization (intracellular) with the LST1 8D12 antibody. A rat antibody of the same isotype was used as a non-specific control (grey curve). 


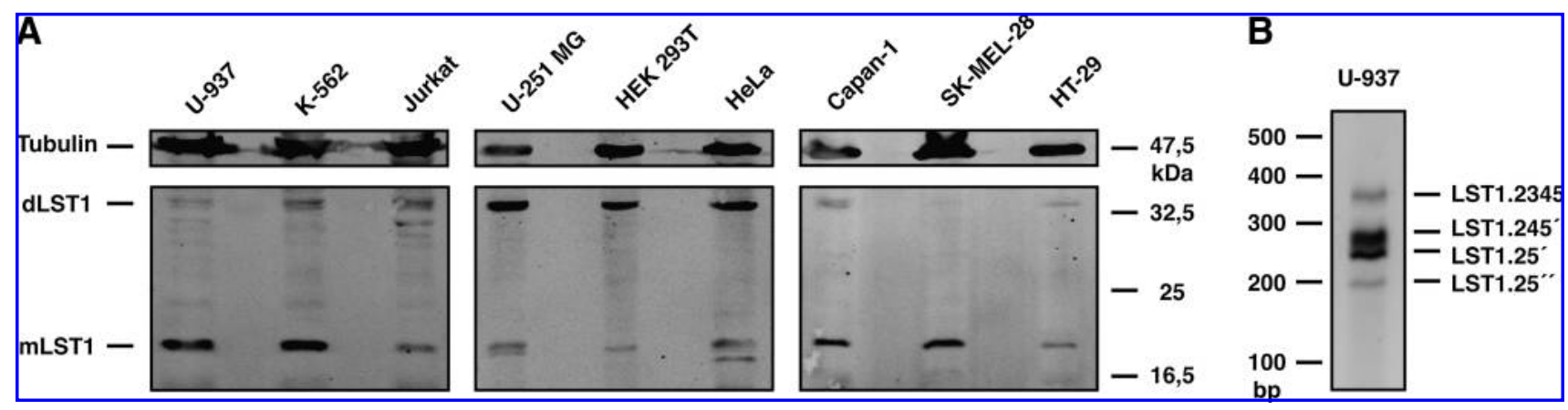

FIG. 3. LST1 expression in human cell lines. (A) Analysis of LST1 protein expression. Lysates were probed by Western blot analysis using the LST1 7E2 antibody. Tubulin was detected as a loading control with the WA-3 antibody (M. Schliwa, AdolfButenandt-Institute, Ludwig-Maximilians-Universität München, Germany). The positions of tubulin and endogenous LST1.2345 in its monomeric (mLST1) and dimeric (dLST1) form are indicated. (B) Expression of LST1 transcripts in U-937 cells. LST1 transcripts were amplified by RT-PCR from U-937 total RNA using primers flanking the longest open reading frame of LST1. Based on their length, four splice variants could be identified (LST1.2345, LST1.245', LST1.25', and LST1.25").

transmembrane LST1.2345 and in the suppression of soluble LST1.245/5'.

\section{Discussion}

In the present study we generated the first monoclonal antibodies against LST1. Characterization of the LST1 7E2 antibody revealed that it detects both recombinant and endogenous LST1 via Western blot analysis, but fails to bind non-denatured LST1 polypeptides (data not shown). The binding epitope was localized to the 13 carboxyterminal amino acids present in the six LST1 isoforms encoded by exon 4 containing splice variants. The LST1 8D12 antibody reacted with native recombinant and endogenous LST1 in both immunoprecipitation and flow cytometry procedures. Immunoprecipitation of recombinant LST1 isoforms revealed that the 8D12 antibody is able to bind at least four LST1 isoforms. Using the LST1 7E2 antibody, we detected LST1 in a number of human cell lines. Expression was not limited to immune cells but was found in all examined cell lines at comparable levels. With both antibodies only the full-length transmembrane isoform LST1.2345 was detected, although all isoforms with internal in-frame deletions are recognized by both antibodies (see Figs. 1A and 2A). The failure to detect endogenous soluble LST1.245/5' is not due to rapid export, as we did not detect any LST1 polypeptides in concentrated cell culture supernatants, even of high-expressing HEK 293T transfectants (data not shown). Only a polypeptide corresponding to the longest LST1 isoform, LST1.2345, was also found by Raghunatan and colleagues probing human dendritic cells with a polyclonal LST1-peptide specific serum. ${ }^{(6)}$ These results stand in contrast to previous findings at the mRNA level, which stated higher amounts and an increase in splice variants in immune cells when compared to cells from nonlymphoid tissues. ${ }^{(2)}$ In all cell lines tested, only the full-length LST1 isoform was detected, although based on mRNA results soluble isoforms (see LST1.245' in Fig. 3B) should predominate. ${ }^{(4)}$ Moreover, HEK 293 T cells transiently transfected with the FLAG-LST1.245 vector regularly displayed a substantially weaker expression than the transmembrane isoforms (Fig. 1A). Thus, shorter LST1 polypeptides are either rapidly degraded or are synthesized at a lower level. It is possible that shorter LST1 molecules need to be stabilized by a partner. The discrepancy between LST1 transcript and protein expression leads us to propose that LST1 expression is tightly regulated at the translational or post-translational level. Previous studies found that in rheumatoid arthritis-affected blood and synovium cells LST1 splice variants encoding soluble isoforms were drastically upregulated. ${ }^{(4)}$ We propose that in inflammation the suppression of soluble isoforms is defeated by transcriptional activation. The monoclonal LST1 antibodies generated and characterized in this study should provide powerful tools for future research into the role of LST1 in inflammatory diseases.

\section{Acknowledgments}

We would like to thank Stefanie Faerberboeck for excellent technical assistance; Anja Gahl for providing the PEI transfection reagent; and the Neuroimmunology Department at the Max Planck Institute of Neurobiology, Martinsried, for the use of the FACSCalibur cytometer. This work was supported by the Helmholtz Zentrum Muenchen doctoral program, research field "Infection and Immunity."

\section{References}

1. de Baey A, Holzinger I, Scholz S, Keller E, Weiss EH, and Albert E: Pvu II polymorphism in the primate homologue of the mouse B144 (LST-1). A novel marker gene within the tumor necrosis factor region. Hum Immunol 1995;42:9-14.

2. de Baey A, Fellerhoff B, Maier S, Martinozzi S, Weidle U, and Weiss EH: Complex expression pattern of the TNF region gene LST1 through differential regulation, initiation, and alternative splicing. Genomics 1997;45:591-600.

3. Holzinger I, de Baey A, Messer G, Kick G, Zwierzina H, and Weiss, EH: Cloning and genomic characterization of LST1: a new gene in the human TNF region. Immunogenetics 1995; 42:315-322.

4. Mulcahy H, O'Rourke KP, Adams C, Molloy MG, and O'Gara F: LST1 and NCR3 expression in autoimmune inflammation and in response to IFN-gamma, LPS and microbial infection. Immunogenetics 2006;57:893-903.

5. Yu X, and Weissman SM: Characterization of the promoter of human leukocyte-specific transcript 1 . A small gene with a 
complex pattern of alternative transcripts. I Biol Chem 2000;275:34597-34608.

6. Rollinger-Holzinger I, Eibl B, Pauly M, Griesser U, Hentges F, Auer B, Pall G, Schratzberger P, Niederwieser D, Weiss EH, and Zwierzina H: LST1: a gene with extensive alternative splicing and immunomodulatory function. J Immunol 2006; 164:3169-3176.

7. Raghunathan A, Sivakamasundari R, Wolenski J, Poddar R, and Weissman SM: Functional analysis of B144/LST1: a gene in the tumor necrosis factor cluster that induces formation of long filopodia in eukaryotic cells. Exp Cell Res 2001;268: 230-244.

8. Nagy GR, Gyõrffy B, Galamb O, Molnár B, Nagy B, and Papp Z: Use of routinely collected amniotic fluid for wholegenome expression analysis of polygenic disorders. Clin Chem 2006;52:2013-2020.
9. Rottach A, Kremmer E, Nowak D, Leonhardt H, and Cardoso MC: Generation and characterization of a rat monoclonal antibody specific for multiple red fluorescent proteins. Hybridoma 2008;27:337-343.

Address correspondence to: Prof. Dr. Elisabeth H. Weiss Biozentrum LMU Grosshadernerstr. 2 82152 Planegg-Martinsried Germany

E-mail: elisabeth.weiss@lmu.de

Received: April 9, 2009 Accepted: May 11, 2009 
This article has been cited by:

1. Christian Schiller, Johanna E. Huber, Kalliope N. Diakopoulos, Elisabeth H. Weiss. 2013. Tunneling nanotubes enable intercellular transfer of MHC class I molecules. Human Immunology 74:4, 412-416. [CrossRef]

2. Tatiana Nedelko, Heike Kollmus, Frank Klawonn, Sabine Spijker, Lu Lu, Manuela Heßman, Rudi Alberts, Robert W Williams, Klaus Schughart. 2012. Distinct gene loci control the host response to influenza H1N1 virus infection in a time-dependent manner. BMC Genomics 13:1, 411. [CrossRef] 